\title{
Residual Stress and Surface Energy of Sputtered TiN Films
}

\author{
Xiaolu Pang, Liqiang Zhang, Huisheng Yang, Kewei Gao, and Alex A. Volinsky
}

(Submitted June 4, 2014; in revised form September 1, 2014; published online January 21, 2015)

\begin{abstract}
Morphology, structure, residual stress, and surface energy of magnetron-sputtered titanium nitride (TiN) thin films, deposited at $300{ }^{\circ} \mathrm{C}$ with a thickness in the $0.5-1.7 \mu \mathrm{m}$ range, were characterized. Film microstructure, the origin of residual stress, and its effect on the surface energy were analyzed. The grain size increased with the film thickness. X-ray diffraction showed (200) to (111) preferred orientation transitions with the increasing film thickness. The stress in the TiN films changed from compressive $-0.3 \mathrm{GPa}$ to tensile with the film thickness reaching $0.3 \mathrm{GPa}$. Larger grain size, initial porosity, and sub-grains generation are the reasons for significant changes in the residual stress. Surface energy was investigated by contact angle of water and glycerol droplets, which both show a significant change with the different stress state and crystal preferred orientation. The TiN films form a contact angle larger than $100^{\circ}$ with water as a test liquid, demonstrating their hydrophobicity. While the residual stress changes from compressive to tensile, the contact angle reaches $118^{\circ}$, and the corresponding surface energy changes from 38.8 to $24.2 \mathrm{~mJ} /$ $\mathrm{m}^{2}$. One can expect to achieve a certain desired surface state of TiN films for potential applications.
\end{abstract}

Keywords residual stress, surface energy, TiN films

\section{Introduction}

Thin TiN films deposited by physical vapor deposition have a wide range of applications in machine components, industrial tools, computer disk drives, precision instruments, and human replacement organs (Ref 1-3). TiN has high hardness, good chemical, and metallurgical stability (Ref 4). However, such films, when grown on rigid substrates, are generally in a stressed state, which influences the film's mechanical performance (Ref 5). Film stress thickness dependence for TiN films was reported, attributed to lower defect density in thicker films (Ref6). Janssen (Ref 5) observed a highly compressive average residual stress for thinner TiN films, which decreased for thicker films, and proved that the actual stress in PVD TiN films is a gradient over the thickness, and that the stress was not relaxed in thicker films. However, in Ref 6, it was not observed that the stress in the TiN films undergoes a change from highly compressive to tensile with increasing thickness. Daniel (Ref 7) and Janssen (Ref 8) reported that sputtered $\mathrm{Cr} / \mathrm{CrN}$ dual films' stress state is strongly affected by the $\mathrm{Cr}$ bonding layer, including its thickness and structure. In Daniel's (Ref 7) experiment, the effect of the ion irradiation on the development of the compressive stress was grain size-, texture- and morphology-dependent compressive stress, and can be controlled by the growth conditions. Similarly, the tensile thermal stress varies with the layer thickness, since the thermal expansion coefficient is a structure-dependent property,

Xiaolu Pang, Xiaolu Pang, Liqiang Zhang, Huisheng Yang, and Kewei Gao, Department of Materials Physics and Chemistry, University of Science and Technology Beijing, Beijing 100083, China; and Alex A. Volinsky, Department of Mechanical Engineering, University of South Florida, Tampa, FL 33620. Contact e-mail: pangxl@mater.ustb.edu.cn. varying with grain size. Based on these results, the average stress, as a sum of basic competing structure-dependent stress components, determines the final stress level in the layer. However, Janssen (Ref 8) showed that the stress in $\mathrm{Cr} / \mathrm{CrN}$ is not uniform over the thickness of the film. High tensile stresses are observed near the substrate-film interface. Lower tensile stresses are observed further away from the interface. Moreover, it has been shown that the tensile stress is generated at the grain boundaries. When the film deposition is accompanied by an ion bombardment, compressive stress is generated. The tensile and compressive stresses in these films are independent and additive, however, these results did not hold for all high melting point films, notably not for TiN.

Surface properties of TiN have been studied a lot experimentally, focusing on their comprehensive applications in different fields, such as bio-implants and anti-corrosion coatings (Ref 9-11). However, only surface oxidation, roughness, morphology, and bio-compatibility were studied. Surface energy, as the most important factor of TiN films surface properties, is lacking detailed experimental studies. However, surface energy studies employing computer simulations have been reported. Marlo and Milman (Ref 12) used density functional calculations to investigate the three surfaces of TiN that occur most commonly: (100), (110), and (111), determining surface relaxation characteristics, surface energies, and the adsorption of molecular hydrogen on TiN (100) surface. Further work of Ciobanu, Tambe and Shenoy (Ref 13), based on similar calculations, established the step formation energetics and geometries of TiN (100) and (110) surfaces. Binding and diffusion energies of adatoms, molecules, and small clusters on TiN (100) and (111) surfaces were studied from the first principles to isolate the key atomistic processes that determine the texture evolution during the growth of polycrystalline TiN layers (Ref 14). Other studies investigated the adsorption of different atomic species on TiN surfaces, such as Au on (100) TiN (Ref 15), O on (100) TiN (Ref 16), and water monomers on TiN (100), (110), and (111) surfaces (Ref 17). In this paper, the stress transition from compression to tension and 
preferred crystal orientation of TiN films is discussed, along with the corresponding mechanism and its effects on the surface energy.

\section{Experimental Details}

TiN films were grown by reactive RF-pulsed magnetron sputter deposition in an industrial PVD system at $300{ }^{\circ} \mathrm{C}$. Singlecrystal silicon substrates (110 crystal orientation, $335-\mu \mathrm{m}$ thick) were moved in front of a 76-mm-diameter titanium target. Nitrogen and argon flow during deposition was $1 \mathrm{sccm}$ and 30 sccm, respectively. The base pressure in the sputtering chamber was below $4.8 \times 10^{-3} \mathrm{~Pa}$, while the deposition pressure was kept at $0.3 \mathrm{~Pa}$. The growing film was ion bombarded by applying a substrate bias voltage of $-80 \mathrm{~V}$; the target power was $300 \mathrm{~W}$, resulting in a deposition rate of $7.9 \mathrm{~nm} / \mathrm{min}$.

Prior to sputtering, substrates were cleaned in acetone and ethanol for $10 \mathrm{~min}$, respectively, and subjected to $10 \mathrm{~min}$ in situ Ar plasma cleaning at $50 \mathrm{~W}$ RF power in order to remove any contaminants on the substrate surface and to activate the surface. The target was cleaned using argon (99.99\% pure) gas discharge plasma for $10 \mathrm{~min}$. Initially, an adhesion layer of $\mathrm{Ti}$ metal was deposited for $10 \mathrm{~min}$ and kept constant in all samples, followed by TiN deposition. Film thickness was controlled by adjusting the deposition time, and ranged from 0.47 to $1.72 \mu \mathrm{m}$.

The surface morphological images were characterized using field emission scanning electron microscopy (FSEM). FESEM images were obtained using the Carl Zeiss Supra 55 FESEM. The acceleration voltage of the cathode was set to $10 \mathrm{kV}$. The working distance was set to $9 \mathrm{~mm}$. Different areas were selected for each specimen. The film structure was examined using Rigalu $\mathrm{D} / \mathrm{max}-\mathrm{RB}$ x-ray diffraction with $\mathrm{Cu}$ source $\left(\lambda_{\mathrm{Cu}-\mathrm{K} \alpha}=1.54184 \AA\right)$ with a power of $12 \mathrm{KW}$. The scanning range of the $2 \theta$ diffraction angle varied from $20^{\circ}$ to $90^{\circ}$ with $0.02^{\circ}$ step size, and $2 \% \mathrm{~min}$ scan speed. Full width at half maximum (FWHM) and crystal size of the films was obtained from measured XRD patterns by means of the ANALYZE program. PDF-2 coupled with Inorganic Crystal Structure Database was used to analyze the crystalline phase content of the samples. The film stress was calculated from the substrate curvature radius change using the Stoney's equation. The substrate profile was measured with Veeco Dektak 150 stylus profilometer. Distilled water and glycerol were used to measure the surface energy by means of the contact angle test with different power and time plasma treatment samples. Liquid droplets $(\sim 3.5 \mu \mathrm{L})$ were placed vertically from the $3-\mathrm{mm}$ height with the micro-injector on each sample. Ten measurements were taken, and the average value was reported.

\section{Results and Discussion}

\subsection{Film Structure}

FSEM micrographs in Fig. 1 show surface microstructure of TiN films with different thickness. The morphology of the TiN films changes significantly with the film thickness. For the $0.47-\mu$ m-thick film, the grain size is very small (Fig. 1a), gradually increase with the film thickness. For the $1.72-\mu \mathrm{m}-$ thick film, small grains appear at grain boundaries, compared
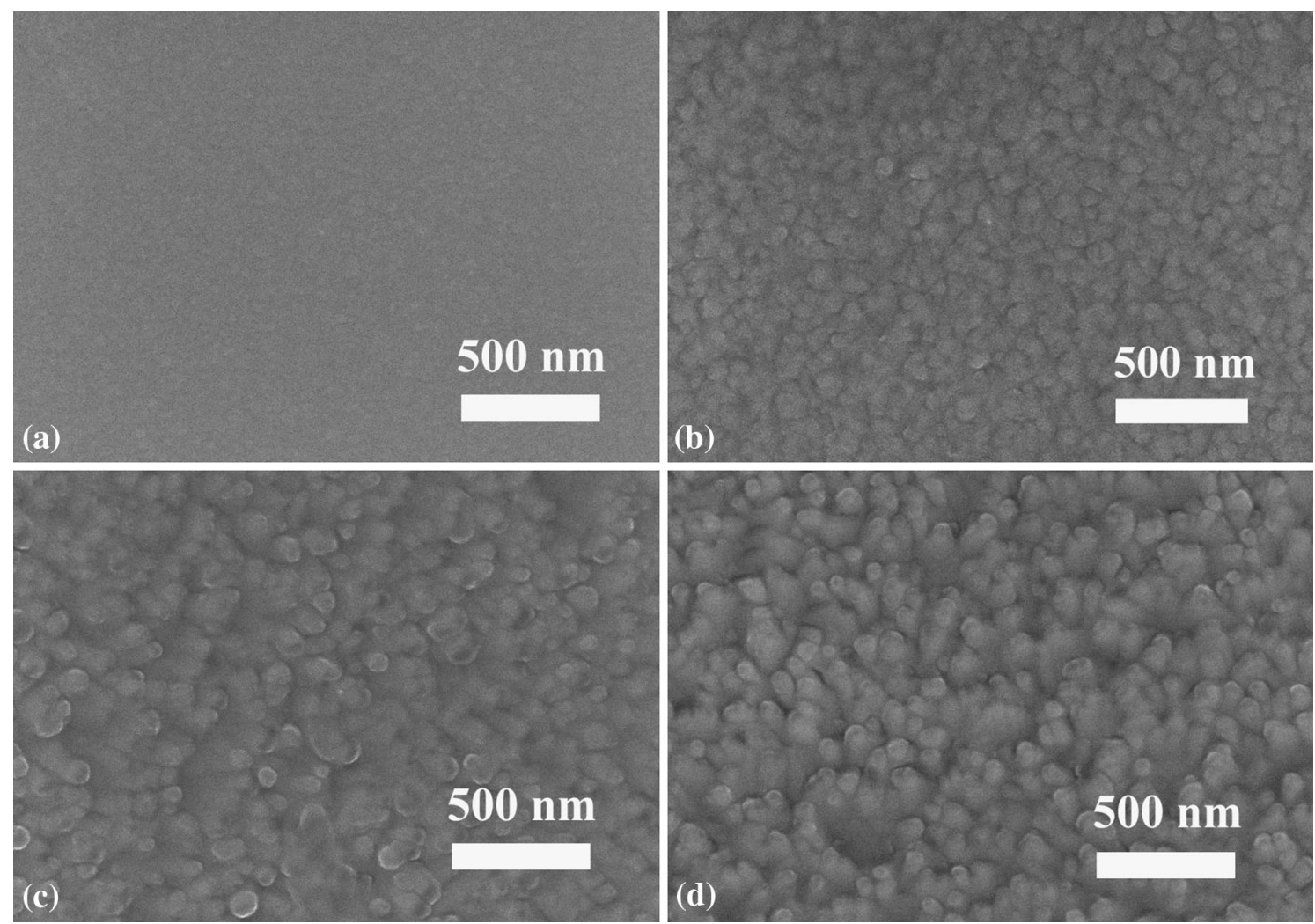

Fig. 1 FSEM micrographs showing the surface morphology of (a) $0.47-\mu \mathrm{m}-$, (b) $0.87-\mu \mathrm{m}-$, (c) $1.30-\mu \mathrm{m}-$, and (d) $1.72-\mu \mathrm{m}-$ thick TiN films 


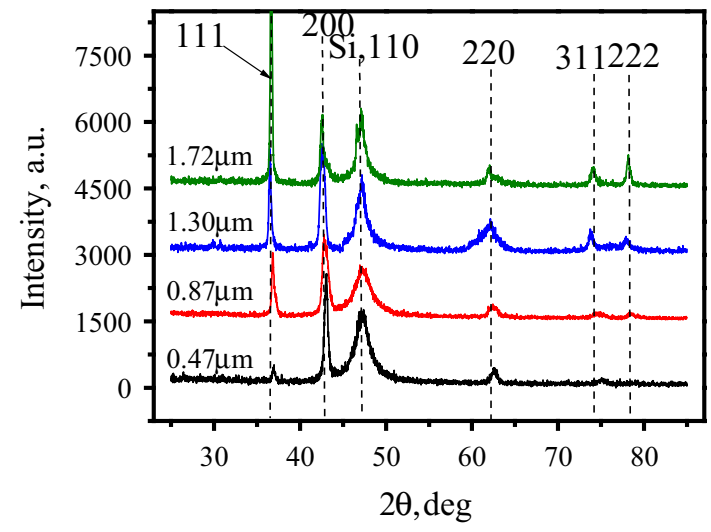

Fig. 2 XRD spectra of different thickness TiN films

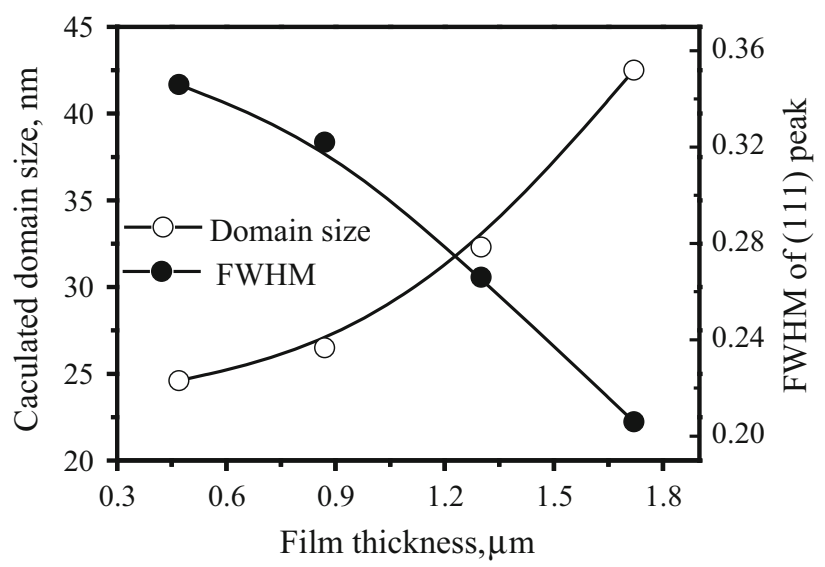

Fig. 3 FWHM of TiN films (111) reflections. Trend lines added for clarity

with thinner films (Fig. 1d). TiN films morphology changed significantly with increasing thickness, which also affects the residual stress.

XRD $\theta / 2 \theta$ scans from reactively magnetron-sputtered TiN layers deposited with increasing thickness on Si (110) substrates are presented in Fig. 2. The diffraction patterns reveal the presence of the FCC mono-phase $\mathrm{B} 1-\mathrm{NaCl}$ structure, showing mainly (111) and (200) reflections. Texture transitions from (200) to (111) preferred orientation with increasing film thickness. Films show (111) preferred orientation with increasing film thickness. The big shift of the peak position to higher $2 \theta$ diffraction angles, with respect to the relaxed structure of randomly oriented FCC TiN in thinner films, indicates in-plane compression in the layers. This result is consistent with the measured residual stress in the films. Figure 3 shows the values of the FWHM of the rocking curve around the (111) diffraction peak. FWHM decreased with the film thickness. As seen in Fig. 3, the FWHM of the $1.72-\mu \mathrm{m}$ film is the narrowest, revealing the best crystalline structure.

The size of the coherently diffracting domains $\langle\mathrm{D}\rangle$ in the films was calculated as (Ref 18)

$\langle D\rangle=\frac{0.9 \lambda}{B \cos \theta}$

Here, $\lambda$ is the XRD wavelength and $\theta$ is the Bragg's angle. Parameter $\langle D\rangle$ corresponds to the average length of crystalline
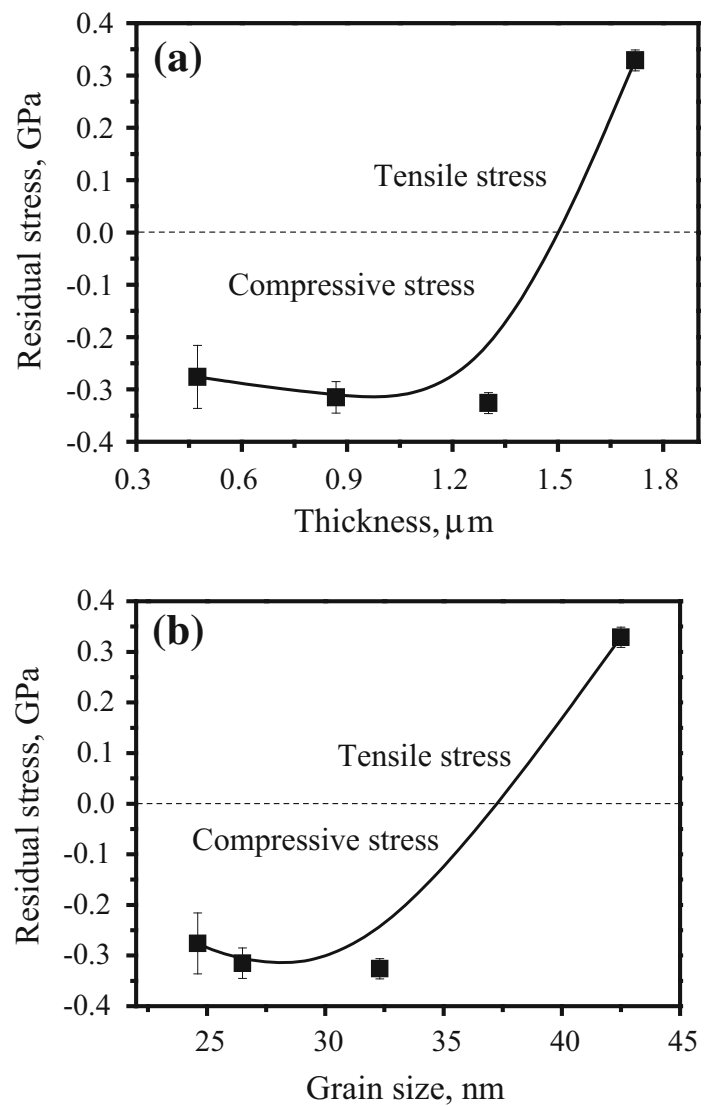

Fig. 4 (a) Average residual stress in TiN films as a function of film thickness; (b) Average residual stress in TiN film as a function of grain size

domains in the direction of the diffraction vector, and represents crystalline regions with no planar defects (Ref 19), while $B$ is the FWHM value. Figure 3 indicates that $\langle D\rangle$ initially increased with the film thickness in the 0.47-1.72 $\mu \mathrm{m}$ range.

\subsection{Residual Stress in TiN Films}

The residual stress in the films, $\sigma$, was calculated using the Stoney's equation (Ref 20):

$\sigma=\frac{1}{6}\left(\frac{1}{R_{\mathrm{a}}}-\frac{1}{R_{\mathrm{b}}}\right) \frac{E}{1-v} \frac{t_{\mathrm{s}}^{2}}{t_{\mathrm{f}}}$

Here, $R_{\mathrm{b}}$ and $R_{\mathrm{a}}$ are radii of the substrate curvature before and after film deposition, respectively, $E$ is the Young's modulus, $v$ is the Poisson's ratio of the substrate, and $t_{\mathrm{s}}$ and $t_{\mathrm{f}}$ are the thicknesses of the substrate and the film, respectively. Substrate profiles were measured using Dektak 150 stylus profilometer. Profiles corresponding to compressive and tensile stresses have positive and negative curvatures, respectively

In Fig. 4(a), the average residual stress is plotted as a function of the film thickness. For the $0.47-\mu \mathrm{m}$ film, $-0.264 \mathrm{GPa}$ compressive stress was calculated, gradually increasing to $-0.34 \mathrm{GPa}$ for the $0.87-\mathrm{nm}$ thick film. Subsequently, compressive stress changes to tensile at $1.72 \mu \mathrm{m}$ film thickness, reaching $0.33 \mathrm{GPa}$. The residual stress trend observed in this paper is different from Ref. 5 and 6. This is likely caused by the adhesive layer and the deposition temperature. 
As seen in Fig. 4(a), the stress in sputtered TiN films can be either compressive or tensile, depending on the film thickness. The transition from compressive to tensile stress can be explained by the following two reasons (Ref 21, 22). First, as the size of crystals increases, the number of defects decreases and films become denser, causing a natural tendency of the film to develop tensile stress with the film thickness. Second, compressive stresses develop in the film by "atomic shot peening", associated with the collision of neutral inert gas atoms (created in the sputtering process) with the growing film.

Compressive stresses can be understood qualitatively in terms of both the implantation of atoms in the film during deposition and the micro-plastic deformation, associated with the atomic collisions. Energetic argon atoms become implanted into the film, resulting in compressive stresses. The excess atoms dilate the material near the surface, which leads to compressive stresses due to the constraint of the underlying film. Also, atomic collisions with the growing surface result in plastic deformation events. Each surface element can be deformed plastically. This deformation would occur by incremental dislocations movement in the film, or other shear processes. Again, the constraint of the underlying film leads to biaxial compression in the film. The tensile stresses present in a sputtered film depend on the tendency of the film to shrink once attached to the substrate. Thus, the stresses that develop are associated with the internal atomic rearrangement or densification of the film. If the sputtered structure relaxes, then intrinsic tensile stresses develop in the film.

At the initial deposition stage, the grain size is very small in the $0.47-0.87 \mu \mathrm{m}$ film thickness range. Compressive stress originates from the coating structure with a high density of point defects. At this stage, attachment to the substrate restrains lattice expansion, significantly contributing to residual compressive stress. Along with increasing film thickness (controlled by sputtering time), grain size increases, and the density of grain boundaries (length per surface area) decreases, which results in reduced porosity in the films. The lower the grain boundaries density, the less possibility exists for the extra atoms to be inserted into the grain boundaries (Ref 23), which, in turn, decreases the compressive stress, transforming it into tensile stress. Chaudhari (Ref 23) pointed out that grain boundaries are less dense than the crystal lattice, so that grain growth (elimination of some grain boundaries) leads to densification of the film and thus to tensile stresses in the film.

In Ref. 24, it was indicated that the increase in the residual stress of the TiN film is caused by the smaller grain size and the transition of the texture from (200) to (111) preferred orientation. This conclusion agrees well with our findings. The relationship between the grain size and the residual stress was also investigated. In this paper, the grain size is equivalent to the size of the coherently diffracting domains $\langle D\rangle$, as shown in Fig. 4(b). This result indicates that TiN films with grain size smaller than $35 \mathrm{~nm}$ exhibit higher compressive stress and above $35 \mathrm{~nm}$ have a tensile stress. Reference 24 also indicates that grain growth generally leads to stress relaxation when the film is under compressive stress. When the initial grain size is below a critical value, grain growth can occur by normal boundary migration, and generates tensile stress in the plane of the film. This result agrees well with our investigation.

\subsection{Surface Energy of TiN Films}

The static contact angles of water and glycerol on TiN films surface were determined with a video-based optical system
OCA 15 . Liquid droplet $(\sim 3.5 \mu \mathrm{L})$ was placed vertically from the $3-\mathrm{mm}$ height with the micro-injector on each sample. Ten measurements were taken, and an average value was reported. The measurements were conducted at room temperature $\left(25^{\circ} \mathrm{C}\right)$.

Figure 5 shows the contact angles of water on TiN films of different thickness. There are no obvious changes in the contact angle for the films between 0.47 - and $1.30-\mu \mathrm{m}$ thick, however, there is a sudden increase with the film thickness of $1.72 \mu \mathrm{m}$. Similar results are also seen in Fig. 6 with glycerol droplets on TiN films. The exact contact angles are shown in Fig. 7 and 8. TiN films' contact angles are $101.3 \pm 0.8,100.7 \pm 2.4$, $100.7 \pm 1.3,118 \pm 2.6$ with water, and $82 \pm 0.5,81 \pm 0.2$, $81.5 \pm 0.3,88.3 \pm 0.1$ with glycerol.

The relationship between the solid surface energy, $\gamma_{S V}$, liquid surface energy, $\gamma_{\mathrm{LV}}$, solid-liquid interface interaction energy, $\gamma_{\mathrm{SL}}$, and the equilibrium contact angle, $\theta \gamma$, in the solid/ liquid/gas three-phase system is known as the Young's equation:

$\gamma_{\mathrm{SV}}=\gamma_{\mathrm{SL}}+\gamma_{\mathrm{LV}} \cos \theta_{\gamma}$

The Owens-Wendt and Kaelble approach (Ref 25, 26) was used to determine the total surface energy, along with the dispersive and the polar components. The thermodynamic work of adhesion is related to the substrate surface energy as:
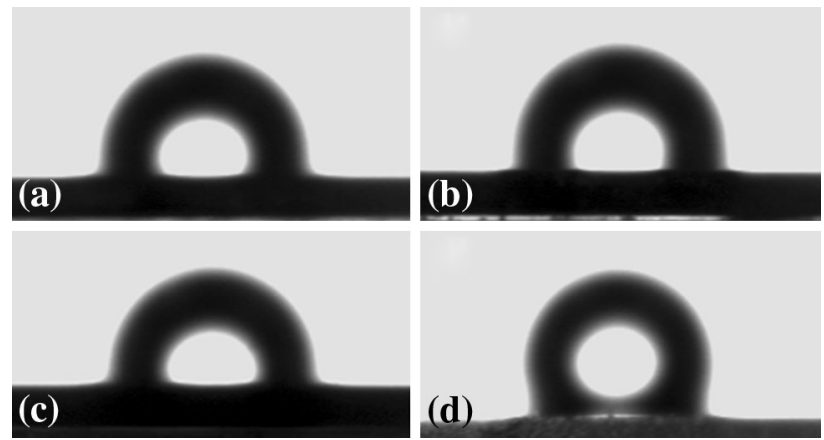

Fig. 5 Optical images of contact angle with water droplets on different thickness TiN films: (a) $0.47 \mu \mathrm{m}$, (b) $0.87 \mu \mathrm{m}$, (c) $1.30 \mu \mathrm{m}$, and (d) $1.72 \mu \mathrm{m}$
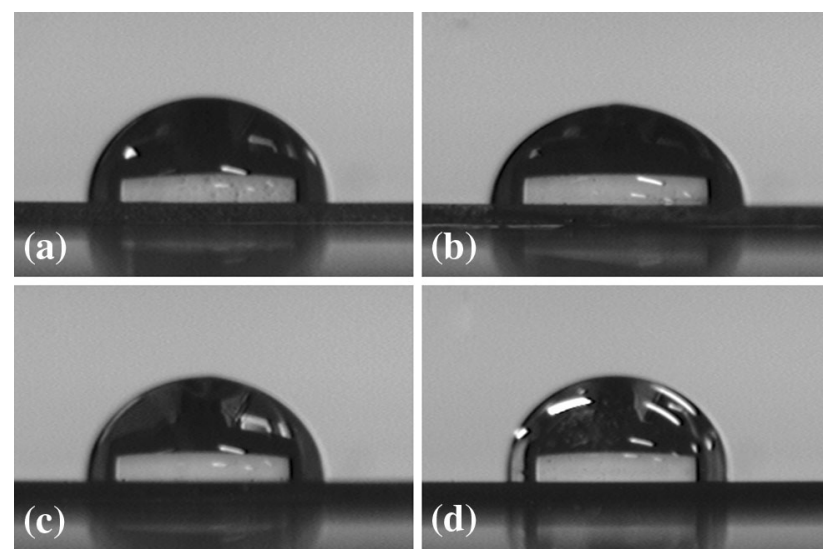

Fig. 6 Optical images of contact angle with glycerol droplets on different thickness TiN films: (a) $0.47 \mu \mathrm{m}$, (b) $0.87 \mu \mathrm{m}$, (c) $1.30 \mu \mathrm{m}$, and (d) $1.72 \mu \mathrm{m}$ 


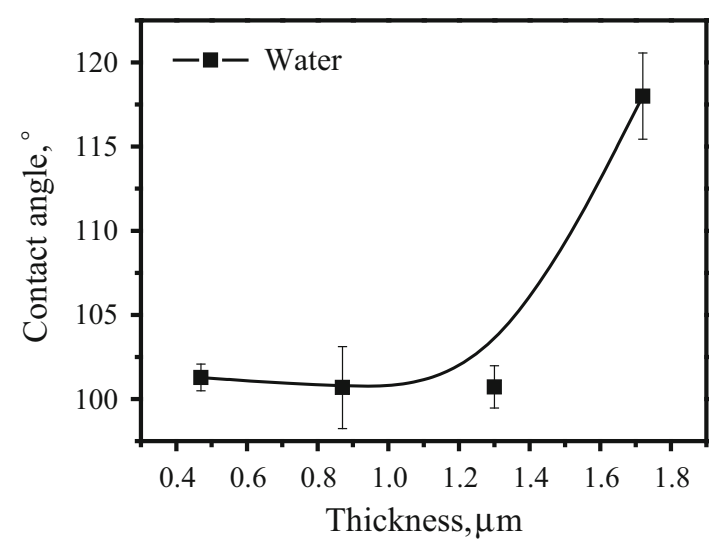

Fig. 7 Contact angle of water droplets on TiN films at different thickness

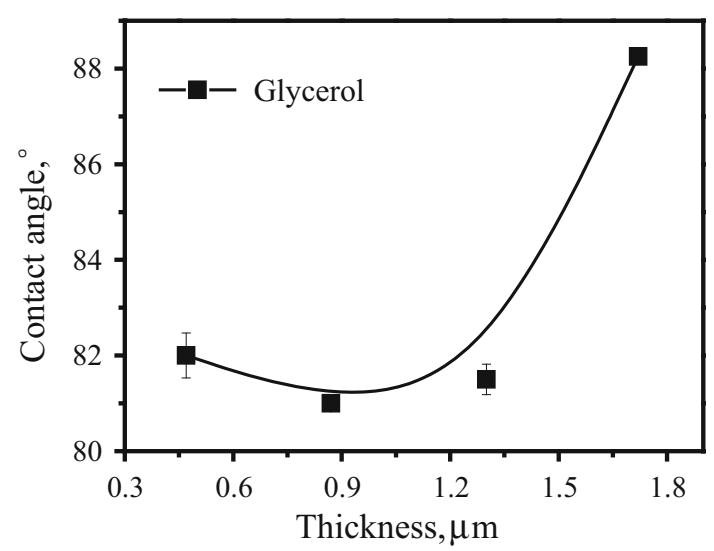

Fig. 8 Contact angle of glycerol droplets on TiN films at different thickness

$W_{\mathrm{a}}=\gamma_{\mathrm{SV}}+\gamma_{\mathrm{LV}}-\gamma_{\mathrm{SL}}$

Combining Eqs 3 and 4 yields:

$W_{\mathrm{a}}=\gamma_{\mathrm{LV}}\left(1+\cos \theta_{\gamma}\right)$

The thermodynamic work of adhesion can also be expressed using dispersive and polar components (Ref 27):

$W_{\mathrm{a}}=2 \sqrt{\gamma_{\mathrm{SL}}^{\mathrm{d}} \gamma_{\mathrm{LV}}^{\mathrm{d}}}+2 \sqrt{\gamma_{\mathrm{SV}}^{\mathrm{p}} \gamma_{\mathrm{LV}}^{\mathrm{p}}}$

Combining Eqs 5 and 6 yields:

$\gamma_{\mathrm{LV}}\left(1+\cos \theta_{\gamma}\right)=2 \sqrt{\gamma_{\mathrm{SL}}^{\mathrm{d}} \gamma_{\mathrm{LV}}^{\mathrm{d}}}+2 \sqrt{\gamma_{\mathrm{SV}}^{\mathrm{p}} \gamma_{\mathrm{LV}}^{\mathrm{p}}}$

To determine the surface energy of a coated sample surface along with the dispersive and polar components of the surface energy, a proper selection of liquids must be made. This should include combination of dispersive and polar liquids with a wide range of surface energy, along with variable and different dispersive and polar components. Thus, distilled water and glycerol were used. Table 1 contains the surface energy data for these liquids.

Surface energy of TiN films can be calculated using the measured components of water and glycerol in Table 1. The value of surface energy only changes slightly for the films with
Table 1 Surface energy components water and glycerol $\left(\mathrm{mJ} / \mathrm{m}^{2}\right)$

\begin{tabular}{llll}
\hline & $\gamma_{\mathbf{L V}}$ & $\gamma_{\mathbf{L V}}^{\mathbf{d}}$ & $\gamma_{\mathbf{L V}}^{\mathbf{p}}$ \\
\hline Water & 72.8 & 21.8 & 51.0 \\
Glycerol & 64 & 34 & 30 \\
\hline
\end{tabular}

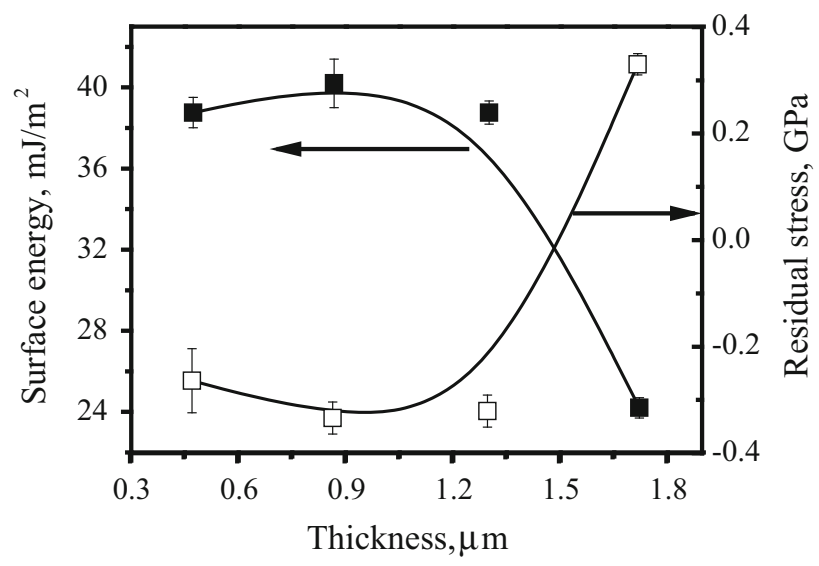

Fig. 9 Surface energy and residual stress change with the film thickness

the thickness of $0.47,0.87$, and $1.30 \mu \mathrm{m}$, which corresponds with $38.8,40$, and $38.7 \mathrm{~mJ} / \mathrm{m}^{2}$, however, the value shows a sharp decrease to $24.2 \mathrm{~mJ} / \mathrm{m}^{2}$ with the $1.72 \mu \mathrm{m}$ thickness. The biggest change in these films is stress, thus the relationship between stress and surface energy is plotted in Fig. 9. The surface energy changes quite a bit when the stress changes from compression to tension. For solids, specific surface energy is not equal to surface tension, since the atoms in solids do not move fast enough. Actually, atoms and/or molecules in solids move extremely slow, such that a solid does not change its shape rapidly. This is why a thin sheet of paper maintains its shape for thousands of years in spite of a large surface area and a high value of the total surface energy. Consequently, a solid surface may be elastically deformed. The number of atoms and/ or molecules per unit surface area of a solid cannot be treated as constant, depending on the degree of deformation. The change in the total surface energy of a solid is due to the change in both total surface area and deformation. Shuttleworth (Ref 28) studied surface stress of solids, which has the same units as surface tension, by modeling a solid surface as a membrane that is coherently connected with the bulk substrate underneath. He considered a membrane with the size of $\alpha \times \alpha$. Under biaxial surface stress, $\sigma$, the membrane changes its size to $(\alpha+\delta \alpha) \times$ $(\alpha+\delta \alpha)$, while changing its area by $\delta \mathrm{A}=2 \alpha \delta \alpha$. The work done by the biaxial surface stress is $\sigma \delta \mathrm{A}$, and the change in the surface energy is $\gamma \delta \mathrm{A}+\mathrm{A} \delta \gamma$. From energy conservation, one has $\sigma \delta \mathrm{A}=\gamma \delta \mathrm{A}+\mathrm{A} \delta \gamma$. Thus, the surface stress in a solid is defined as:

$\sigma=\gamma+\frac{\partial \gamma}{\partial \varepsilon}$

where $\varepsilon \equiv \delta A / A$ denotes the surface strain.

If the films show compressive stress, the value of $\frac{\partial \gamma}{\partial \varepsilon}$ is negative, and $\gamma$ should be much larger than the tensile stress, which is consistent with our experimental results in Fig. 9. 


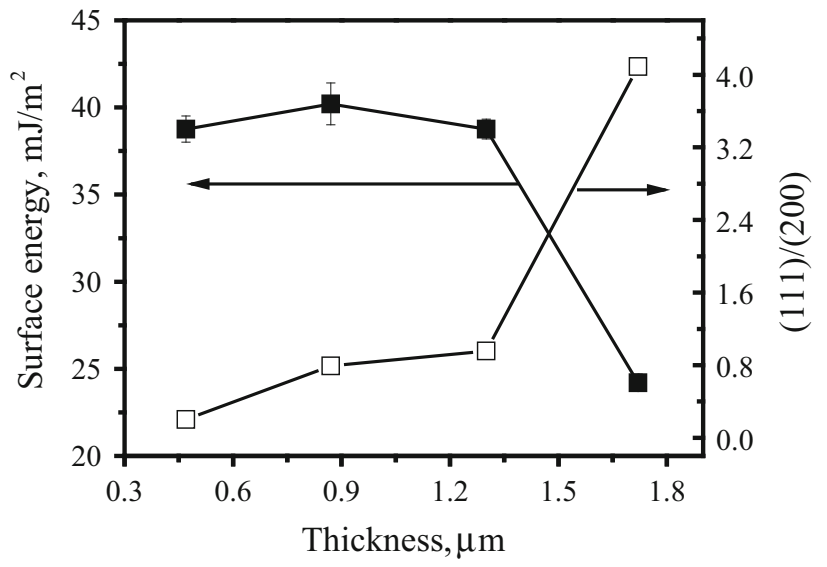

Fig. 10 Surface energy and crystal preferred orientation change with the film thickness

Figure 10 shows the relationship between the preferred orientation and the surface energy. When the ratio of (111)/ (200) is smaller than 0.8 , no obvious changes in surface energy were observed. However, with the ratio of $(111) /(200)$ increasing to 4 , the surface energy shows a sharp decrease. Using the nearest-neighbor interaction approximation, the binding energy for one mole of atoms, $E_{\mathrm{b}}$, in a three-dimensional FCC crystal is given by

$\gamma=\frac{E_{\mathrm{b}}}{4 N_{A}}\left(\frac{N}{A}\right)$

where $N / A$ is the number of atoms per unit area. For the (111) plane, $\left(\frac{N}{A}\right)_{(111)}=\frac{4}{a_{0}^{2} \sqrt{3}}$, where $a_{0}$ is the bulk lattice constant. Thus,

$\gamma_{(111)}=\frac{E_{\mathrm{b}}}{N_{A} a_{0}^{2} \sqrt{3}}$

For the (200) plane in the FCC crystal, there are four broken bonds per atom and the number of atoms per unit area is $\left(\frac{N}{A}\right)_{(200)}=\frac{4}{a_{0}^{2}}$. Thus,

$\gamma_{(200)}=\frac{E_{\mathrm{b}}}{N_{A} a_{0}^{2}}$

From Eqs 10 and 11, it is obvious that the surface energy of TiN films with the (111) preferred orientation will be lower than with the (200) orientation, which is consistent with the experimental results shown in Fig. 10. On the other hand, with the film growth, the natural driving force is to minimize the surface energy, so the surface energy dominates the preferred orientation. Accordingly, the preferred orientation is determined by the critical competition between the strain and the surface energy. One can expect to be able to fabricate TiN films with desired surface states for certain applications.

\section{Conclusions}

In this work, film structure, the origin of residual stress and the effect of residual stress on surface energy were studied in sputtered TiN films of different thickness. Grain size increases with the film thickness, while thick films are porous. For the $1.72-\mu \mathrm{m}$ thick film, sub-grains appear at grain boundaries, and structure gets denser. The stress in the TiN films undergoes a change from compressive $(-0.264 \mathrm{GPa})$ to tensile $(0.33 \mathrm{GPa})$ with increasing film thickness. The development of compressive stress in the film at the initial stage by "atomic shot peening" is associated with the collision of neutral inert gas atoms (created in the sputtering process) with the growing film. However, tensile stresses developed in sputtered films at a later stage, and depend on the tendency of the film to shrink once it is attached to the substrate and cooled down to room temperature. Surface energy shows a significant change with the different stress states and preferred crystal orientation. The TiN films form a contact angle larger than $100^{\circ}$ with water as a test liquid, which demonstrates that the TiN film surface is hydrophobic. When the residual stress changes from compressive to tensile, the contact angle goes up to $118^{\circ}$, corresponding to the surface energy change from 38.8 to $24.2 \mathrm{~mJ} / \mathrm{m}^{2}$. When the ratio of the $(111) /(200)$ intensities is smaller than 0.8 , there are no obvious changes in the surface energy. However, when the ratio of the $(111) /(200)$ intensities increases to 4 , the surface energy shows a sharp decrease, which means that preferred orientation and stress may influence the surface energy to an expected value for certain applications.

\section{Acknowledgments}

This work was supported by the National Nature Science Foundation of China (51271022) and the Fok Ying Tung Education Foundation (132001). AV acknowledges support from the National Science Foundation under the IRES:1358088 Grant.

\section{References}

1. K. Holmberg, H. Ronkainen, K. Holmberg, and A. Laukkanenl, A Model for Stresses, Crack Generation and Fracture Toughness Calculation in Scratched TiN-Coated Steel Surfaces, Wear, 2003, 254, p 278-291

2. X. Pang, H. Yang, K.W. Gao, Y. Wang, and A.A. Volinsky, AlTiN Layer Effect on Mechanical Properties of Ti-Doped Diamond-Like Carbon Composite Coatings, Thin Solid Films, 2011, 519, p 53535357

3. R. Machunze and G.C.A.M. Janssen, Stress and Strain in Titanium Nitride Thin Films, Thin Solid Films, 2009, 517, p 5888-5893

4. F.J. Espinoza-Beltrán, O. Che-Soberanis, L. Garciá-González, and J. Morales-Hernández, Effect of the Substrate Bias Potential on Crystalline Grain Size, Intrinsic Stress and Hardness of Vacuum Arc Evaporated TiN/c-Si Coatings, Thin Solid Films, 2003, 437, p 170-175

5. R. Machunze and G.C.A.M. Janssen, Stress Gradients in Titanium Nitride Thin Films, Surf. Coat. Technol., 2008, 203, p 550-553

6. H. Köstenbauer, G.A. Fontalvo, M. Kapp, J. Keckes, and C. Mitterer, Annealing of Intrinsic Stresses in Sputtered TiN Films: The Role of Thickness-Dependent Gradients of Point Defect Density, Surf. Coat. Technol., 2007, 201, p 4777-4780

7. R. Daniel, K.J. Martinschitz, J. Keckes, and C. Mitterer, The Origin of Stresses in Magnetron-Sputtered Thin Films with Zone $T$ Structures, Acta Mater., 2010, 58, p 2621-2633

8. G.C.A.M. Janssen, Stress and Strain in Polycrystalline Thin Films, Thin Solid Films, 2007, 515, p 6654-6664

9. B.-H. Moon, H.-C. Choe, and W.A. Brantley, Surface Characteristics of TiN/ZrN Coated Nanotubular Structure on the Ti-35Ta-x Hf Alloy for Bio-Implant Applications, Appl. Surf. Sci., 2012, 258, p 2088-2092

10. B. Subramanian, C.V. Muraleedharan, R. Ananthakumar, and M. Jayachandran, A Comparative Study of Titanium Nitride (TiN), Titanium Oxy Nitride (TiON) and Titanium Aluminum Nitride 
(TiAlN), As Surface Coatings for Bio Implants, Surf. Coat. Technol., 2011, 205, p 5014-5020

11. A. Kumar and D. Kaur, Nanoindentation and Corrosion Studies of TiN/ NiTi Thin Films for Biomedical Applications, Surf. Coat. Technol., 2009, 204, p 1132-1136

12. M. Marlo and V. Milman, Density-Functional Study of Bulk and Surface Properties of Titanium Nitride Using Different ExchangeCorrelation Functionals, Phys. Rev. B, 2000, 62, p 2899-2907

13. C.V. Ciobanu, D.T. Tambe, and V.B. Shenoy, First-Principles Calculations of Step Formation Energies and Step Interactions on TiN (001), Surf. Sci., 2005, 582, p 145-150

14. D. Gall, S. Kodambaka, M.A. Wall, I. Petrov, and J.E. Greene, Pathways of Atomistic Processes on TiN (001) and (111) Surfaces During Film Growth: An Ab Initio Study, J. Appl. Phys., 2003, 93, p 9086

15. N.C. Hernandez and J.F. Sanz, A First Principles Density Functional Study of Au Deposition on TiN (001) Surface, Int. J. Mol. Sci., 2001, 2, p 263-270

16. J. Graciani, J.F. Sanz, T. Asaki, K. Nakamura, and J.A. Rodriguez, Interaction of Oxygen with TiN (001): $\mathrm{N} \leftrightarrow \mathrm{O}$ Exchange and Oxidation Process, J. Chem. Phys., 2007, 126, p 244713-244720

17. S. Sanyal, U.V. Waghmare, and J.A. Ruud, Adsorption of Water on TiN (100) (110) and (111) Surfaces: A First-Principles Study, Appl. Surf. Sci., 2011, 257, p 6462-6467

18. K. Kusaka, T. Ao, T. Hanabusa, and K. Tominaga, Effect of External Magnetic Field on $c$-Axis Orientation and Residual Stress in AIN Films, Thin Solid Films, 1998, 332, p 247-251
19. H. Köstenbauer, G.A. Fontalvo, J. Keckes, and C. Mitterer, Intrinsic Stresses and Stress Relaxation in TiN/Ag Multilayer Coatings During Thermal Cycling, Thin Solid Films, 2008, 516, p 1920-1924

20. G.C.A.M. Janssen, M.M. Abdalla, F. van Keulen, B.R. Pujada, and B. van Venrooy, Celebrating the 100th Anniversary of the Stoney Equation for Film Stress: Developments from Polycrystalline Steel Strips to Single Crystal Silicon Wafers, Thin Solid Films, 2009, 517, p 1858-1867

21. J. Xiong, W. Qin, X. Cui, B. Tao, J. Tang, and Y. Li, ThicknessInduced Residual Stresses in Textured YBCO Thin Films Determined by Crystalline Group Method, Phys. C, 2007, 455, p 52-57

22. P.R. Guduru, E. Chason, and L.B. Freund, Mechanics of Compressive Stress Evolution During Thin Film Growth, J. Mech. Phys. Solids, 2003, 51, p 2127-2148

23. P. Chaudhart, Grain Growth and Stress Relief in Thin Films, J. Vac. Sci. Technol., 1972, 9, p 520-522

24. C.-L. Chang, J.-Y. Jao, W.-Y. Ho, and D.-Y. Wang, Effects of TitaniumImplanted Pre-treatments on the Residual Stress of TiN Coatings on High-Speed Steel Substrates, Surf. Coat. Technol., 2007, 201, p 67026706

25. D.K. Owens and R.C. Wendt, Estimation of the Surface Free Energy of Polymers, J. Appl. Polym. Sci., 1969, 13, p 1741-1747

26. D.H. Kaelble, Dispersion-Polar Surface Tension Properties of Organic Solids, J. Adhesion, 1970, 2, p 66-81

27. W.A. Zisman, Influence of Constitution on Adhesion, Ind. Eng. Chem., 1963, 55, p 18-38

28. R. Shuttleworth, The Surface Tension of Solids, Proc. Phys. Soc. A, 1950, 63, p 444-457 